\title{
RESISTÊNCIA DE GENÓTIPOS DE BATATA (Solanum spp.) A Diabrotica speciosa (GERMAR, 1824) (COLEOPTERA: CHRYSOMELIDAE)
}

\author{
RESISTANCE OF POTATOES GENOTYPES (Solanum spp.) TO Diabrotica speciosa (GERMAR,1824) \\ (COLEOPTERA: CHRYSOMELIDAE)
}

\author{
Fernando Mesquita Lara ${ }^{1}$ Marcelo Poletti ${ }^{2}$ José Carlos Barbosa $^{3}$
}

\section{RESUMO}

\begin{abstract}
Avaliou-se a resistência de genótipos de batata (Solanum spp.) a Diabrotica speciosa (Germar,1824) em condições de campo e laboratório. O plantio de campo foi efetuado em julho de 1997, na FCAV/ UNESP, Campus de Jaboticabal, utilizando-se os seguintes genótipos: Achat, NYL 235-4, 288.759-16, 288.771-6, 288.776-3, 288.776-6, 288.787-5, 288.801-8 e 288.814-7. Avaliaram-se o consumo de área foliar em levantamentos realizados aos 70 e 90 dias após plantio e os danos ocasionados pelas larvas aos tubérculos, após a colheita. Em laboratório, foram realizados testes de preferência para alimentação com plantas de 40 dias (teste sem chance de escolha), envolvendo os nove genótipos presentes no campo, e com plantas de 47 e 68 dias (com e sem chance de escolha), avaliando-se cinco genótipos. Constatou-se, no ensaio de campo, que os genótipos 288.759-16, 288.776-3, 288.776-6, 288.787-5 e 288.814-7 foram menos consumidos por adultos de D. speciosa que a cultivar Achat, porém, em laboratório, 288.759-16 e 288.776-6 não revelaram essa característica. O consumo em NYL 235-4 e 288.801-8 foi intermediário, não diferindo dos demais. NYL 2354 e 288.801-8 foram resistentes às larvas, apresentando, em média, menos que 3 furos por tubérculo. O genótipo 288.776-3 destacou-se como resistente a larvas e adultos de D. speciosa sendo a resistência a adultos do tipo não-preferência para alimentação (antixenose).
\end{abstract}

Palavras-chaves: insecta, resistência varietal, antixenose, preferência alimentar.

\section{SUMMARY}

The resistance of potato genotypes to Diabrotica speciosa (Germar,1824) (Coleoptera: Chrysomelidae), was evaluated under field and laboratory conditions. The field trial was set up in the experimental farm of FCAV - UNESP, Jaboticabal, SP, and the following genotypes were compared:
Achat, NYL 235-4, 288.759-16, 288.771-6, 288.776-3, 288.776-6, 288.787-5, 288.801-8 e 288.814-7. The leaf area consumption by adults was evaluated at 70 and 90 days after planting, and, after harvest, larval damage to tubers (number of perforations) was assessed. In the laboratory, feeding preference trials were conducted 40 days after plant emergence in no choice tests, for all nine genotypes, and at 47 and 68 days, in no choice and free choice tests, using five select genotypes. In the field test, the genotypes 288.759-16, 288.776-3, 288.776-6, 288.787-5e 288.814-7 were less attacked by adults. However, 288.759-16 and 288.776-6 did not show this performance under laboratory conditions. NYL 235-4 and 288.801-8 were resistant to larval tuber damage. The genotype 288.776-3 showed resistance to larvae and adults of Diabrotica speciosa, and feeding nonpreference was the resistance mechanism to the adults.

Key words: Insecta, host plant resistance, antixenosis, feeding preference.

\section{INTRODUÇÃO}

A cultura da batata (Solanum tuberosum L.) tem enfrentado graves problemas de queda na produção, que pode atingir $33 \%$ devido ao ataque de insetos (FRANÇA \& BARBOSA, 1987). Para evitar esse prejuízo, o gasto com controle químico pode chegar a $19 \%$ do custo de produção (CURZIO, 1993).

MAGALHÃES \& CARVALHO (1988) mencionam Diabrotica speciosa (Germar, 1824) como praga principal ou secundária de várias culturas, incluindo solanáceas, e que se encontra difundi-

\footnotetext{
${ }^{1}$ Engenheiro Agrônomo, Professor Titular, Depto de Fitossanidade, Faculdade de Ciências Agrárias e Veterinárias (FCAV), Universidade Estadual Paulista (UNESP), Rodovia Carlos Tonanni, km 5, 14870-000, Jaboticabal, SP. E-mail: fmlara@fcav.unesp.br. autor para correspondência.

${ }^{2}$ Acadêmico do curso de Agronomia, FCAV/UNESP.

${ }^{3}$ Engenheiro Agrônomo, Professor Adjunto, Depto de Ciências Exatas, FCAV/UNESP.
} Recebido para publicação em 02.09.99. Aprovado em 22.03.00 
da por todo o território brasileiro. As larvas desse inseto efetuam danos nos tubérculos e nas plantas, na fase de brotação e emergência (FERRO \& BOITEAU, 1993). Os adultos alimentam-se das folhas e possuem hábito constante de voar de uma planta para outra. Dependendo da população, os danos são consideráveis, porque diminuem a área foliar e, conseqüentemente, a produção. Em ensaio simulando injúrias de insetos através da remoção de folhas, CRANSHAW \& RADCLIFFE (1980) verificaram que, quando ocorre desfolha de até $33 \%$ no início do ciclo da cultura, a planta consegue uma boa recuperação, porém, se a mesma for mais intensa, atingindo $67 \%$ ou mais, provocará perdas na produção.

Um dos métodos alternativos de controle desses insetos é o uso de plantas resistentes, que oferece uma série de vantagens, inclusive a de se integrar perfeitamente em programas de manejo de pragas (LARA, 1991). Neste contexto, na década de 40, iniciou-se nos EUA um programa de melhoramento de batata visando à resistência ao crisomelídeo Leptinotarsa decemlineata Say, a partir da batata selvagem mexicana Solanum demissum Lindl. e outras espécies selvagens (TORKA, 1950), como $\boldsymbol{S}$. berthaultii Hawkes, que tem sido utilizada com freqüência em vários programas de desenvolvimento de plantas resistentes de batata a $L$. decemlineata (FLANDERS et al., 1992, FRANÇA \& TINGEY, 1994, FRANÇA $\boldsymbol{e}$ t al., 1994). Essa espécie vem sendo utilizada na Universidade de Cornell, como fonte de resistência a vários insetos e ácaros, levando a bons resultados, inclusive com seus híbridos obtidos com $\boldsymbol{S}$. tuberosum (GREGORY et al., 1986, TINGEY, 1991; PLAISTED et al., 1992). Seguindo essa linha, PLAISTED et al. (1992) produziram o clone NYL 235-4 com boas qualidades agronômicas e alta resistência a $L$. decemlineata. Esse clone sofre três a sete vezes menos perdas na produção do que materiais suscetíveis.

SARGO et al. (1998), comparando a resistência de genótipos de batata a $\boldsymbol{D}$. speciosa, verificaram que NYL 235-4 apresenta um bom potencial agrícola, pois além de ter alta produtividade apresenta resistência a essa praga, sendo 2 a 3 vezes menos atacada por adultos e 4 a 7 vezes menos danificada pelas larvas, em relação a diversas cultivares comerciais suscetíveis.

BONINE (1997) avaliou os danos de larvas nos estolões e em tubérculos de cinco cultivares de batata, efetuando infestações com ovos e larvas de D. speciosa obtidas em laboratório. Verificou maior suscetibilidade da cultivar Baronesa e melhor desempenho da cultivar Macaca, com menor número de estolões danificados, e Santo Amor, com menor número de perfurações nos tubérculos.
A presente pesquisa objetivou avaliar a resistência de genótipos de batata, oriundos dos EUA e/ou melhorados pela Embrapa - Centro Nacional de Pesquisa de Hortaliças (CNPH), ao crisomelídeo Diabrotica speciosa, em condições de campo, e a preferência alimentar de adultos, em laboratório.

\section{MATERIAL E MÉTODOS}

Ensaio em condições de campo: Efetuou-se o plantio em campo (cultivo de inverno) em julho de 1997, na Fazenda de Ensino e Pesquisa da FCAV/UNESP, Jaboticabal, SP, utilizando-se um delineamento em blocos ao acaso, contendo nove tratamentos e quatro repetições. A área útil de cada parcela foi de duas ruas de $2,5 \mathrm{~m}$, espaçadas de $0,70 \mathrm{~m}$, com seis plantas por rua $(0,5 \mathrm{~m}$ entre plantas), perfazendo um total de $3,5 \mathrm{~m}^{2}$. As parcelas foram separadas lateralmente por uma rua e longitudinalmente por duas plantas da cultivar Achat como bordadura, sendo todo ensaio delimitado pela mesma. Cada bloco constou dos seguintes tratamentos: Achat (cultivar comercial), NYL 235-4, 288.759-16, 288.771-6, 288.776-3, 288.776-6, 288.787-5, 288.801-8 e 288.814-7.

As avaliações do consumo de área foliar pelos adultos do inseto foram realizadas aos 70 e 90 dias pós-plantio, coletando-se um folíolo por planta, em 10 plantas amostradas ao acaso, na área útil de cada parcela. Como critério para amostragem, a coleta foi realizada em folhas do terço médio, tomando-se sempre o folíolo do lado direito da folha, situado no terceiro par em relação à base. A área foliar consumida pelos insetos foi determinada com um medidor de área foliar (Li-Cor).

A avaliação dos danos ocasionados pelas larvas foi realizada pós-colheita, contando-se o número de furos por tubérculo, em 10 tubérculos escolhidos ao acaso, dentre os de tamanho médio previamente selecionados em cada parcela.

\section{Ensaios em condições de laboratório:}

Efetuou-se um teste de preferência para alimentação, sem chance de escolha, utilizando-se os nove genótipos presentes no campo, com plantas de 40 dias após o plantio. Através desse primeiro ensaio, com cinco repetições, foram selecionados cinco genótipos: Achat, NYL 235-4, 288.776-3, 288.776-6 e 288.814-7. Com esses, foram realizados dois ensaios. Tanto no primeiro, realizado com folíolos de plantas aos 47 dias após o plantio, como no segundo, utilizando-se folíolos de plantas aos 68 dias, procederam-se testes de preferência para alimentação com e sem chance de escolha.

Nos testes com chance de escolha, discos de folíolos de cada material, com 2,0cm de diâmetro, foram colocados em placas de Petri em círculo, sobre papel filtro umedecido, liberando-se a propor- 
ção de dois adultos por disco no interior de cada placa, e deixando-os se alimentarem até que em um dos materiais o consumo aparente atingisse aproximadamente $75 \%$ da área do folíolo, momento em que os insetos foram retirados, procedendo-se, a seguir, a avaliação do peso úmido consumido (em mg).

Paralelamente foram mantidos discos de folíolos de cada genótipo, nas mesmas condições, mas sem a presença de insetos, que também foram pesados ao final do teste, a fim de corrigir o peso consumido, em função de possível perda de água. Esses ensaios foram realizados em delineamento inteiramente casualisado, com 10 repetições.

Os testes sem chance de escolha, com cinco repetições, foram conduzidos da mesma maneira que o anterior, utilizando-se, porém, apenas um dos materiais por placa de Petri, colocando-se dois discos no interior de cada uma, e quatro insetos por placa.

Todos os ensaios foram mantidos em BOD a $25 \pm 2^{\circ} \mathrm{C}$, na ausência de luz, para se evitar qualquer possível influência da posição das lâmpadas.

Os dados de todos os ensaios foram submetidos à análise de variância pelo teste $\mathrm{F}$, sendo os dados originais transformados em $\mathrm{x}^{1 / 2}$, e as médias comparadas pelo teste de Tukey, em nível de $5 \%$ de probabilidade de erro.

\section{RESULTADOS E DISCUSSÃO}

Ensaio em condições de campo: Os dados de consumo de área foliar por folíolo, devido ao ataque de adultos $\boldsymbol{D}$. speciosa (Tabela 1), mostram que aos 70 dias pós-plantio os genótipos NYL 2354, 288.759-16, 288.776-3, 288.776-6 e 288.787-5 apresentaram danos inferiores a $50,00 \mathrm{~mm}^{2}$ por folíolo, destacando-se o genótipo 288.776-6, no qual o consumo foi de $14,90 \mathrm{~mm}^{2}$ por folíolo. O material que sofreu maior dano foi Achat, que teve uma área consumida superior a $100,00 \mathrm{~mm}^{2}$ por folíolo. Os genótipos 288.771-6, 288.801-8 e 288.814-7 apresentaram dados intermediários.

Aos 90 dias após o plantio, Achat continuou se revelando o mais suscetível, apresentando área foliar consumida superior a $100,00 \mathrm{~mm}^{2}$ por folíolo, e os genótipos 288.759-16, 288.776-3, 288.776-6, e 288.814-7 apresentaram-se como os mais resistentes, com áreas consumidas inferiores a $20 \mathrm{~mm}^{2}$, destacando-se o genótipo 288.776-3, com consumo de $7,10 \mathrm{~mm}^{2}$ por folíolo.

Considerando-se a média das duas avaliações, nota-se que os genótipos 288.759-16, 288.7763 , 288.776-6, 288.787-5 e 288.814-7 revelaram-se
Tabela 1 - Área foliar média consumida por D. speciosa em genótipos de batata, em duas avaliações, e danos de larvas nos tubérculos. Jaboticabal, SP, 1997.

\begin{tabular}{|c|c|c|c|c|}
\hline \multirow[b]{2}{*}{ Genótipos } & \multicolumn{3}{|c|}{ Área foliar $\left(\mathrm{mm}^{2} \text { por folíolo }\right)^{1}$} & \multirow{2}{*}{$\begin{array}{c}\mathrm{N}^{0} \text { de furos/ } \\
\text { tubérculo }^{1}\end{array}$} \\
\hline & 70 Dias & 90 Dias & Média & \\
\hline ACHAT & $114,00 \mathrm{a}$ & $107,00 \mathrm{a}$ & $110,50 \mathrm{a}$ & $7,54 \mathrm{ab}$ \\
\hline NYL 235-4 & $17,80 \mathrm{~b}$ & $87,00 \mathrm{ab}$ & $51,20 \mathrm{ab}$ & $2,74 \mathrm{c}$ \\
\hline $288.759-16$ & $6,40 \mathrm{~b}$ & $17,50 \mathrm{c}$ & $12,00 \mathrm{~b}$ & $4,57 \mathrm{abc}$ \\
\hline $288.771-6$ & $54,30 \mathrm{ab}$ & $22,40 \mathrm{bc}$ & $38,40 \mathrm{~b}$ & $3,06 \mathrm{bc}$ \\
\hline $288.776-3$ & $25,30 \mathrm{~b}$ & $7,10 \mathrm{c}$ & $16,20 \mathrm{~b}$ & $4,50 \mathrm{abc}$ \\
\hline $288.776-6$ & $14,90 \mathrm{~b}$ & $8,50 \mathrm{c}$ & $11,70 \mathrm{~b}$ & $2,56 \mathrm{c}$ \\
\hline $288.787-5$ & $30,00 \mathrm{~b}$ & $27,20 \mathrm{bc}$ & $33,90 \mathrm{~b}$ & $5,92 \mathrm{abc}$ \\
\hline $288.801-8$ & $65,60 \mathrm{ab}$ & $33,20 \mathrm{bc}$ & $49,40 \mathrm{ab}$ & $2,39 \mathrm{c}$ \\
\hline $288.814-7$ & $60,90 \mathrm{ab}$ & $11,60 \mathrm{c}$ & $36,30 \mathrm{~b}$ & $7,71 \mathrm{a}$ \\
\hline F (Tratamentos) & $4,99 *$ & $7,75 *$ & $0,45 *$ & $5,15 *$ \\
\hline $\mathrm{CV}$ & 39,82 & 37,63 & 27,07 & 20,56 \\
\hline
\end{tabular}

${ }^{1}$ Dados originais. * Significativo em nível de $5 \%$ de probabilidade. ${ }^{\text {ns }}$ Não significativo. Médias seguidas de mesma letra não diferem entre si pelo teste de Tukey, em nível de 5\% de probabilidade de erro.

resistentes aos adultos de $\boldsymbol{D}$. speciosa, destacando-se entre eles 288.776-6 e 288.759-16, nos quais os insetos consumiram $11,70 \mathrm{~mm}^{2}$ e $12,00 \mathrm{~mm}^{2}$ respectivamente, enquanto Achat foi o material mais suscetível $\left(110,50 \mathrm{~mm}^{2}\right)$.

Em relação aos danos causados pelas larvas alfinete (Tabela 1), verifica-se que os genótipos NYL 235-4, 288.776-6 e 288.801-8 apresentaram-se como os mais resistentes, com 2,74, 2,56, e 2,39 furos/tubérculo, enquanto 288.814-7 foi o mais suscetível, com 7,72 furos/tubérculo. Os demais materiais em teste revelaram danos intermediários. Com respeito ao NYL 235-4, SARGO et al. (1998), em ensaio semelhante para avaliação de resistência às larvas, em Jaboticabal, SP, também destacaram esse genótipo como um dos menos danificados por larvas de D. speciosa, quando comparado a diversas cultivares comerciais. STEIN (1998) constatou que esse clone portou-se como resistente a pragas de solo, na região de Campinas, SP.

\section{Ensaios em condições de laboratório:}

Os resultados do primeiro teste, sem chance de escolha, aos 40 dias pós-plantio (Figura 1) revelam que o genótipo menos consumido foi 288.776-3 (21,64mg), enquanto 288.776-6 e 288.759-16 foram os mais consumidos $(136,02 \mathrm{mg}$ e $125,02 \mathrm{mg}$, respectivamente). Nos demais genótipos, os insetos apresentaram consumo intermediário.

Nos ensaios realizados com os cinco genótipos selecionados a partir do ensaio anterior (Tabela 2), nota-se que no teste com chance de escolha, 


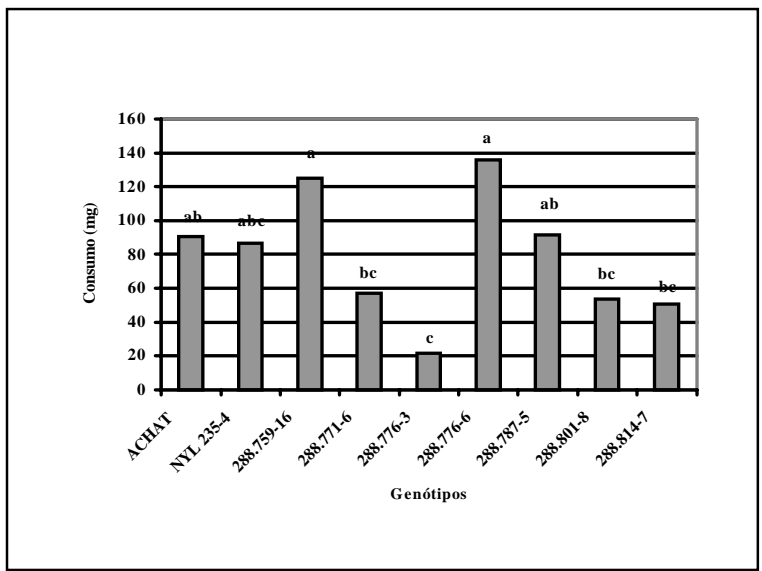

Figura 1 - Consumo (peso matéria fresca - mg) de D. speciosa em discos de folíolos de genótipos de batata (40 dias pósplantio) em teste sem chance de escolha. Jaboticabal, SP, 1997. impede qualquer conclusão a respeito da resistência desse material.

\section{CONCLUSÕES}

O genótipo 288.776-3 é resistente a adultos e larvas de D. speciosa; a sua resistência a adultos é do tipo não-preferência para alimentação (antixenose). NYL 235-4 e 288.801-8 são resistentes ao ataque das larvas aos tubérculos. A cultivar Achat é suscetível a adultos e larvas desse inseto.

\section{AGRADECIMENTOS}

Os autores expressam seus agradecimentos ao CNPq, à FAPESP, a The McKnight Foundation e ao Centro de Pesquisas de Hortaliças/EMBRAPA, pelos auxílios financeiros e bolsas. aos 47 dias, não houve diferença significativa entre os dados. Já no teste sem chance de escolha (47 dias), verificouse menor preferência do inseto pelo genótipo 288.776-3 (42,4mg) e maior consumo em 288.776-6 (99,00mg).

Aos 68 dias pós-plantio, tanto no ensaio com chance de escolha como no sem chance, o genótipo 288.776-3 apresentou-se como o mais resistente, ou seja, menos preferido para alimentação, revelando resultado semelhante ao observado aos 47 dias. Constatou-se maior preferência do inseto pelo genótipo 288.776-6, apesar de não ter diferido significativamente da cultivar 288.814-7, no teste com chance de escolha.

Comparando-se os dados dos testes de campo, consumo de área foliar $\left(\mathrm{mm}^{2}\right)$ e resistência às larvas (dano nos tubérculos), com os ensaios realizados em condição de laboratório, observa-se que o genótipo 288.776-6, apesar de ter sido pouco atacado em condições de campo, apresentou-se como o mais preferido para alimentação em laboratório. Portanto, sugere-se a ocorrência de pseudo-resistência no ensaio de campo, provavelmente devido à baixa infestação da praga relacionada com outros fatores não avaliados, como porte da planta, número de folhas etc, que podem ter interferido no desempenho desse material frente aos demais. Deve-se ressaltar também que, provavelmente, o baixo ataque das larvas aos tubérculos desse genótipo tenha sido decorrência da baixa infestação na parte aérea, o que
Tabela 2 - Consumo (peso matéria fresca - mg) de D. speciosa em discos de folíolos de genótipos de batata em teste com e sem chance de escolha. Jaboticabal, SP, 1997.

\section{Consumo (mg) ${ }^{1}$}

\begin{tabular}{lcccc} 
Genótipos & \multicolumn{2}{l}{ Teste com Chance de Escolha } & \multicolumn{2}{l}{ Teste sem Chance de Escolha } \\
\cline { 2 - 5 } & 47 Dias & 68 Dias & 47 Dias & 68 Dias \\
& & & & \\
& 17,80 & $41,58 \mathrm{ab}$ & $64,04 \mathrm{ab}$ & $94,02 \mathrm{ab}$ \\
ACHAT & 32,46 & $36,46 \mathrm{ab}$ & $75,08 \mathrm{ab}$ & $123,16 \mathrm{ab}$ \\
NYL 235-4 & 28,23 & $21,83 \mathrm{~b}$ & $42,40 \mathrm{~b}$ & $82,40 \mathrm{~b}$ \\
288.776-3 & 34,61 & $59,89 \mathrm{a}$ & $99,00 \mathrm{a}$ & $154,66 \mathrm{a}$ \\
$288.776-6$ & 26,99 & $49,62 \mathrm{a}$ & $65,64 \mathrm{ab}$ & $142,06 \mathrm{ab}$ \\
$288.814-7$ & $1,52 \mathrm{~ns}$ & $3,99 *$ & $4,09 *$ & $3,83 *$ \\
& 25,74 & 29,55 & 16,86 & 16,69 \\
F (Tratamentos) & &
\end{tabular}

Dados originais. *Significativo em nível de $5 \%$ de probabilidade. ${ }^{\text {ns }}$ Não significativo. Médias seguidas de mesma letra não diferem entre si pelo teste de Tukey, em nível de $5 \%$ de probabilidade de erro.

\section{REFERÊNCIA BIBLIOGRÁFICA}

BONINE, D.P. Suscetibilidade de cultivares de batata (Solanum tuberosum L.) a Diabrotica speciosa (Germar) (Coleoptera: Chrysomelidae) e ocorrência de outras pragas subterrâneas. Pelotas, RS, 1997. 59p. Dissertação (Mestrado em Agronomia - Fitossanidade) - Curso de Pósgraduação em Agronomia, Universidade Federal de Pelotas, 1997.

CRANSHAW, W.S., RADCLIFFE, E.B. Effect of defoliation on yield of potatoes. J ournal of Economic Entomology, v.73, p.131-134, 1980.

CURZIO, I. Produzindo mais e melhor. Sinal Verde, v.12, p.26, 1993. 
FERRO, D.N., BOITEAU, G. Management of insect pests. In: ROWE, R. C. (ed.) Potato health management. Wooster: APS, 1993. p.103-116.

FLANDERS, K.L., HAWKES, J.G., RADCLIFFE, $\boldsymbol{e}$ t al. Insect resistance in potatoes: sources, evolutionary relationships, morphological and chemical defenses, and ecogeographical associations. Euphytica, v.61, p.83-111, 1992.

FRANÇA, F.H., BARBOSA, S. O controle de pragas da batata. In: REIFSCHENEIDER, F.J.B.(ed). Produção de batata Brasília : EMBRAPA-CNPH, 1987. p.73-84.

FRANÇA, F.H., TINGEY, W.M. Influence of light level on perfomance of the colorado potato beetle on Solanum berthaultii L. and on resistance expression in S. berthaultii Hawkes. Journal of American Society Horticultural Science, v.119, p.915-919, 1994

FRANÇA, F.H., PLAISTED, R.L., ROUSH, R.T., VIA, S., et al. Selection response of the colorado potato beetle for adaptation to the resistant potato, Solanum berthaultii. Entomologia Experimentalis et Applicata, v.73, p.101-109, 1994.

GREGORY, P., TINGEY, W.M., AVÉ, D.A., et al. Potato glandular trichomes: a physicochemical defensive mechanism against insects. In: GREEN, M.B., HEDIN, P.A. (eds) Natural resistance of plants to pests: roles of allelochemicals. Washington : A.C.S., 1986. p.160-167. (ACS Symposium series, 296).

LARA, F.M. Princípios de resistência de plantas a insetos. 2.ed. São Paulo: Icone, 1991. 336p.
MAGALHÃES, B.P., CARVALHO, S.M. Insetos associados à cultura. In: ZIMMERMANN, M.J. DE O., ROCHA, M., YAMADA, T. (eds.). Cultura do feijoeiro - fatores que afetam a produtividade. Piracicaba : Associação Brasileira para pesquisa da Potassa e do Fosfato, 1988. p.573-589.

PLAISTED, R.L., TINGEY, W.M., STEFFENS, J.C. The germplasm release of NYL.235-4, a clone with resistance to the colorado potato e beetle. American Potato Journal, v.69, p.843-846, 1992.

SARGO, H.L.B., LARA, F.M., POLETTI, M. Resistência de genótipos de batata (Solanum spp.) a Diabrotica speciosa Germar (Coleoptera, Chrysomelidae). In: CONGRESSO BRASILEIRO DE ENTOMOLOGIA, 17, 1998, Rio de Janeiro, RJ. Resumos... Rio de Janeiro : Sociedade Entomológica do Brasil, 1998. v.1. 710p. p.29.

STEIN, C.P. Resistência de cultivares de Solanum tuberosum e de híbridos de $S$. tuberosum x $S$. berthaultii a Myzus persicae (Sulzer), Liriomyza huidobrensis (Blanchard), Phthorimaea operculella (Zeller) e pragas de solo. Piracicaba, SP, 1998. 74p. Tese (Doutorado em Ciências Entomologia) - Curso de Pós-graduação em Agronomia, Universidade de São Paulo, 1998.

TINGEY, W.M. Potato glandular trichomes defensive activity against insect attack. In: HEDIN, P.A. (ed.). Naturally occurring pest bioregulators. Washington : A.C.S., 1991. p.126-135. (ACS Symposium series, 449).

TORKA, M. Breeding potatoes with resistance to the colorado beetle. American Potato Journal, v.27, p.263-271, 1950.

Ciência Rural, v. 30, n. 6, 2000. 